\title{
THE FUKUI MATRIX: A SIMPLE APPROACH TO THE ANALYSIS OF THE FUKUI FUNCTION AND ITS POSITIVE CHARACTER
}

\author{
Patrick Bultinck ${ }^{*}$, , Dorien Clarisse ${ }^{1}$, Paul W. Ayers ${ }^{2}$, Ramon Carbo-Dorca ${ }^{3}$
}

1. Department of Inorganic and Physical Chemistry, Ghent University, Krijgslaan 281 (S3), 9000 Gent, Belgium. Patrick.Bultinck@UGent.be Tel. +3292644423, Fax +3292644983

2. Department of Chemistry, McMaster University, Hamilton, Ontario L8S 4M1, Canada.

3. Institute of Computational Chemistry, University of Girona, Campus de Montilivi, 17071 Girona, Catalonia, Spain

TOC TEXT

The Fukui function is generalized to the Fukui matrix revealing new hitherto not available information on its properties.

\footnotetext{
ABSTRACT

The Fukui matrix is introduced as the derivative of the one-electron reduced density matrix with respect to a change in the number of electrons under constant external potential. The Fukui matrix extends the Fukui function concept: the diagonal of the Fukui matrix is the Fukui function. Diagonalizing the Fukui matrix gives a set of eigenvectors, the Fukui orbitals, and
} 
accompanying eigenvalues. At the level of theory used, there is always one dominant eigenvector, with eigenvalue very close to 1 . The remaining eigenvectors are either zero or come in pairs with eigenvalues of the same magnitude but opposite sign. Analysis of the frontier molecular orbital coefficient in the eigenvector with eigenvalue 1 gives information on the quality of the frontier molecular orbital picture. The occurrence of negative Fukui functions can be easily interpreted in terms of the nodal character of the dominant eigenvector with respect to the remaining eigenvectors and eigenvalues.

KEYWORDS: Fukui function, conceptual DFT

\section{INTRODUCTION}

Chemical reactions are of utmost importance not only to chemistry but also to society as they form a cornerstone of modern welfare through the development of e.g., drugs for lifethreatening diseases. It is therefore no wonder that models explaining molecular reactivity have been created for decades and continue to be developed. One of the best-known models is that based on the Frontier Molecular Orbitals (FMO) [1]. Although developed before most chemists became familiar with Density Functional Theory (DFT) and hence before the field known as Conceptual DFT existed, FMO theory can be connected to conceptual DFT provided some (common) approximations are accepted. The same is true for the Hard and Soft Acids and Bases (HSAB) theory [2] which was also embedded in conceptual DFT [3-6]. The energy of a molecule can be written as a functional of the number of electrons $N$ and the external potential $v(\mathbf{r})$ [7]. 
In chemical reactions both of these entities change for each reaction partner because the approach of the other reactant changes the external potential and induces charge transfer. The energy of a molecule can be expressed as follows:

$$
\begin{aligned}
E[v(\mathbf{r}), N]= & E\left[v^{0}(\mathbf{r}), N^{0}\right] \\
& +\left(\frac{\partial E[v(\mathbf{r}), N]}{\partial N}\right)_{\substack{v^{0}(\mathbf{r})=v(\mathbf{r}) \\
N=N^{0}}}\left(N-N^{0}\right)+\frac{1}{2}\left(\frac{\partial^{2} E[v(\mathbf{r}), N]}{\partial N^{2}}\right)_{\substack{v^{0}(\mathbf{r})=v(\mathbf{r}) \\
N=N^{0}}}\left(N-N^{0}\right)^{2}+\cdots \\
& +\int\left(\frac{\delta E[v(\mathbf{r}), N]}{\delta v(\mathbf{r})}\right)_{\substack{v(\mathbf{r})=v^{0}(\mathbf{r}) \\
N=N^{0}}} \delta v(\mathbf{r}) d \mathbf{r}+\cdots \\
& +\frac{1}{2} \int\left(\frac{\partial \delta E[v(\mathbf{r}), N]}{\partial N \delta v(\mathbf{r})}\right)_{\substack{v(\mathbf{r})=v^{0}(\mathbf{r}) \\
N=N^{0}}} d N \delta v(\mathbf{r}) d \mathbf{r}+\cdots
\end{aligned}
$$

The energy is a functional of the external potential $v(\mathbf{r})$ and the number of electrons $N$ and (1) allows computing the energy in terms of both these variables, with self-explanatory notation. The derivatives in (1) all have significance in so-called conceptual DFT and the reader is referred to Geerlings et al. for a review [8]. In this paper we focus on the first mixed derivative which, using the Hellmann Feynman theorem, can be expressed as:

$\left(\frac{\partial \delta E[v(\mathbf{r}), N]}{\partial N \delta v(\mathbf{r})}\right)_{\substack{N=N^{0} \\ v(\mathbf{r})=v^{0}(\mathbf{r})}}=\left(\frac{\partial \rho(\mathbf{r})}{\partial N}\right)_{v_{v(\mathbf{r})=v^{0}(\mathbf{r})}}=f(\mathbf{r})$

$\rho(\mathbf{r})$ is the electron density of the molecule and $f(\mathbf{r})$ is the so-called Fukui function [9-13] which depends on the position coordinate but obviously also on the number of electrons in the system. The Fukui function is one of the most often used reactivity descriptors and is mainly 
used for understanding nucleophilic/electrophilic molecular reactivity. Due to the discontinuity in the energy versus the number of electrons, one cannot truly take the derivative and one must distinguish a right or upper limit and left or lower, giving rise to two different Fukui functions [9-13]. This leads to the working equations:

$f^{+}(\mathbf{r})=\left[\rho_{N+1}(\mathbf{r})-\rho_{N}(\mathbf{r})\right]_{v^{0}(\mathbf{r})=v(\mathbf{r})}$

and

$f^{-}(\mathbf{r})=\left[\rho_{N}(\mathbf{r})-\rho_{N-1}(\mathbf{r})\right]_{v^{0}(\mathbf{r})=v(\mathbf{r})}$

The limitation of constant external potential means keeping the molecular geometry and atom types fixed.

In this work we generalize the Fukui function by introducing a Fukui matrix. This allows us to address two questions: 1. "Can one assess the quality of a frontier orbital approach in a simple way?" 2. "Can one rationalize the occurrence of negative Fukui functions?". Both questions deserve attention. Chemical reactivity is often interpreted in terms of the FMO, even though this is not always appropriate. There is a continuing interest in negative Fukui functions [14-22], and especially how they can be rationalized, and whether they have chemical significance.

The relation between the Fukui matrix and the Fukui function is reminiscent of the relation between the density matrix and the density function. This analogy also immediately makes clear why the introduction of the Fukui matrix is important. Just as with density matrices and electron density functions where the impossibility of physically non-meaningful negative 
electron densities is most apparent from the fact that having natural orbitals with negative occupation numbers violates N-representability [23], the possibility of regions in space with negative Fukui functions may be investigated through the eigenvalues of the Fukui matrix. Moreover, the introduction of a Fukui matrix allows the introduction of orthonormal Fukui orbitals for correlated wave functions. The two questions introduced above are also highly relevant for workers in conceptual DFT as they often rely on FMO and the Fukui matrix reveals whether this is a reasonable approximation.

\section{THEORETICAL METHODS}

Although the Hohenberg-Kohn theorem promises that the electron density suffices to compute all information about a molecule [24], not all expectation values can be computed in a practical way from the electron density. The density matrix often gives more accessible information or allows for a simpler calculation of expectation values. In this work, we introduce the Fukui matrix in such a way that, like the relationship of the electron density to the density matrix [25], the Fukui function appears as the diagonal $\left(\mathbf{r}=\mathbf{r}^{\prime}\right.$ in (5)). The Fukui matrix is therefore given as:

$$
f\left(\mathbf{r}, \mathbf{r}^{\prime}\right)=\left(\frac{\partial \rho\left(\mathbf{r}, \mathbf{r}^{\prime}\right)}{\partial N}\right)_{v(\mathbf{r})=v^{0}(\mathbf{r})}
$$

A similar idea was introduced by Balawender and Geerlings using ensemble density matrices [26]. However, its properties were not discussed as deeply as in the present paper. We focus on the properties of this matrix, its spectral decomposition in a relevant basis and its 
importance for chemical reactivity. In the rest of this paper, we work in a basis-set representation instead of the spatial representation. Specifically, we express the density matrix in the basis of the natural orbitals $\varphi$ of the neutral molecule, as obtained from any ab initio calculation:

$$
P_{i j}=\left\langle\varphi_{i}(\mathbf{r})\left|\rho\left(\mathbf{r}, \mathbf{r}^{\prime}\right)\right| \varphi_{j}\left(\mathbf{r}^{\prime}\right)\right\rangle
$$

So the Fukui matrix projected on this basis is defined as:

$$
\mathbf{f}=\left\{f_{i j}=\left(\frac{\partial P_{i j}}{\partial N}\right)_{v^{0}(\mathbf{r})=v(\mathbf{r})}\right\}
$$

To compute the Fukui matrix as the difference of two density matrices with different number of electrons, we need to express both density matrices in the same basis. The framework for the Fukui matrix remains the same for correlated wave functions but as we want to examine the quality of the FMO approximation, we work at the single determinant level of theory where to every molecular orbital an orbital energy is associated. At the Hartree-Fock level (and formally also the Kohn-Sham level) all matrices can be expressed in terms of the (real) molecular orbitals of the neutral molecule, which in this study is always a closed shell singlet. At the currently used level of theory, the density matrix of the neutral molecule in terms of the molecular orbitals is diagonal, although it is more general and more convenient from an algebraic standpoint to consider the entire matrices. The transformation of the molecular orbitals from the ionic species is a straightforward unitary transformation that requires only the expansion coefficient matrices of the molecular orbitals in the basis set that is common to both calculations. Using $\mathbf{C}$ and $\mathbf{D}$ to respectively denote these matrices for the neutral and ionized molecule, the two Fukui matrices can be easily obtained as: 


$$
\begin{aligned}
& f_{i j}^{-}=\left(P_{i j}^{N}-\sum_{k} \sum_{m}\left(\mathbf{C}^{-1} \mathbf{D}\right)_{i k} P_{k m}^{N-1}\left(\mathbf{C}^{-1} \mathbf{D}\right)_{m j}^{T}\right) \\
& f_{i j}^{+}=\left(-P_{i j}^{N}+\sum_{k} \sum_{m}\left(\mathbf{C}^{-1} \mathbf{D}\right)_{i k} P_{k m}^{N+1}\left(\mathbf{C}^{-1} \mathbf{D}\right)_{m j}^{T}\right)
\end{aligned}
$$

where superscripts in the density matrix $\mathbf{P}=\left\{P_{i j}\right\}$ refer to the density matrix of the neutral $N$ electron molecule or the charged species with $N-1$ or $N+1$ electrons. Owing to the normalization of the Fukui function, the trace of both Fukui matrices must be 1. The next step in the analysis is the diagonalization of the Fukui matrix $\mathbf{f}^{ \pm}$with elements as in (8) and (9), yielding the decomposition:

$\mathbf{f}^{ \pm}=\sum_{i} \phi_{i} F_{i i}^{ \pm} \phi_{i}^{T}$

Henceforth, the $\phi_{i}$ are called the Fukui orbitals and the $F_{i i}^{ \pm}$are called the Fukui eigenvalues. These Fukui orbitals are expressed as linear combinations of the molecular orbitals of the neutral molecule and the expansion coefficients together with the Fukui eigenvalue thus give immediate information on how much a specific Fukui orbital contributes to the Fukui matrix and thus Fukui function, and which molecular orbitals play the most important role. Given the current interest in spin resolved conceptual DFT [27-31], in our actual calculations, we distinguish two Fukui matrices, one for the $\alpha$ spin electrons and one for the $\beta$ spin electrons.

The representation in equation (10), allows for a very simple assessment of the two basic questions asked above: 
1. If the FMO approximation is perfect, a single eigenvalue equal to 1 should appear and the corresponding Fukui orbital should be composed solely of the FMO of the neutral compound. All other eigenvalues should be exactly zero if one wants to reduce the study of chemical reactivity to the properties of the FMO.

2. A necessary, but not sufficient, condition for a negative Fukui function to appear in some region of the molecule is that there must be at least one negative eigenvalue. Using the Fukui matrix, one can then easily explain how negative Fukui function can arise.

These principles will be investigated for a small test set of molecules.

\section{COMPUTATIONAL METHODS}

In order to answer the two questions raised, B3LYP $6-311++\mathrm{G}(2 \mathrm{~d}, 2 \mathrm{p})$ calculations, including geometry optimization, have been performed on the set of fairly small molecules used previously by Otero et al. in their analysis of negative atom condensed Fukui functions [22]. The neutral molecules are closed shell singlets and all ionic species are taken to have spin multiplicity 2. The Fukui matrix is computed according to equations (8)-(9). By convention, the most abundant electron spin is always $\alpha$ in our calculations. To assess the quality of the algebraic manipulations, we checked that for adding an electron the $\alpha$ Fukui matrix has trace 1 and the $\beta$ part has trace zero and vice versa for removal of an electron. All calculations have been carried out using Gaussian-03 [32] and our own routines for the required algebraic manipulations based on information from formatted checkpoint files. 


\section{RESULTS AND DISCUSSION}

For all 18 molecules, the Fukui matrices have been diagonalized for both the addition of an electron and the removal of an electron. In all cases, the traces of the $\alpha$ and $\beta$ Fukui matrices equal either 1 or 0 with deviations in the order of $10^{-9}$. Interestingly, the Fukui matrix with trace 1, always showed one eigenvalue/vector combination with eigenvalue equal to 1 (henceforth referred to as the dominant Fukui orbital) and many with much smaller eigenvalues. There are several significant positive and negative secondary eigenvalues among the latter that, within numerical accuracy, come in pairs with values of equal magnitude but opposite sign. The Fukui matrices with trace zero lack the eigenvector with eigenvalue 1 but do exhibit a set of paired eigenvalues of equal magnitude but opposite sign. In the following we restrict ourselves to the study of the spin Fukui matrix with trace 1. This corresponds to the $\alpha$ Fukui matrix in case of addition of an electron and the $\beta$ Fukui matrix for removal of an electron.

Table 1 gives the most negative eigenvalue found, the number of positive and negative eigenvalues for all molecules (considering only eigenvectors with eigenvalue above $10^{-4}$ or below $-10^{-4}$ ) as well as the coefficient of the appropriate FMO (HOMO or LUMO of the neutral molecule depending on the process considered) in the eigenvector with eigenvalue 1.

\begin{tabular}{|l|l|l|l|l|l|l|l|l|}
\hline & $\mathbf{f}^{-}$ & & & & $\mathbf{f}^{+}$ & & & \\
\hline
\end{tabular}




\begin{tabular}{|c|c|c|c|c|c|c|c|c|}
\hline Molecule & Pos & Neg & Low & CFMO & Pos & Neg & Low & CFMO \\
\hline $\mathrm{CH}_{2} \mathrm{NH}$ & 8 & 7 & -0.0805 & 1.0000 & 8 & 7 & -0.0187 & 0.9582 \\
\hline $\mathrm{CO}_{2}$ & 11 & 10 & -0.0791 & 1.0000 & 9 & 8 & -0.0146 & 0.9761 \\
\hline $\mathrm{HCN}$ & 7 & 6 & -0.0744 & 1.0000 & 7 & 6 & -0.0221 & 0.9687 \\
\hline $\mathrm{N}_{2} \mathrm{O}$ & 11 & 10 & -0.0719 & 0.9997 & 10 & 9 & -0.0219 & 0.9801 \\
\hline $\mathrm{CO}$ & 7 & 6 & -0.0552 & 0.9998 & 8 & 7 & -0.0658 & 0.8906 \\
\hline $\mathrm{SO}_{2}$ & 15 & 14 & -0.0571 & 0.9999 & 16 & 15 & -0.0861 & 0.9958 \\
\hline $\mathrm{SO}_{3}$ & 16 & 15 & -0.0431 & 1.0000 & 18 & 17 & -0.0367 & 0.9768 \\
\hline $\mathrm{SCN}^{-}$ & 14 & 13 & -0.0841 & 0.9995 & 9 & 8 & -0.0251 & 0.9782 \\
\hline $\mathrm{NCO}^{-}$ & 11 & 10 & -0.0753 & 0.9999 & 9 & 8 & -0.0157 & 0.9865 \\
\hline $\mathrm{NO}_{2}{ }^{-}$ & 12 & 11 & -0.0572 & 0.9998 & 13 & 12 & -0.0514 & 0.9969 \\
\hline $\mathrm{HNO}$ & 8 & 7 & -0.0569 & 0.9999 & 9 & 8 & -0.0174 & 0.9970 \\
\hline NOF & 9 & 8 & -0.0745 & 0.9996 & 10 & 9 & -0.0331 & 0.7145 \\
\hline $\mathrm{CH}_{3} \mathrm{COCH}_{3}$ & 16 & 15 & -0.1060 & 0.9994 & 13 & 12 & -0.0167 & 0.9748 \\
\hline $\mathrm{CH}_{3} \mathrm{COOH}$ & 16 & 15 & -0.1060 & 0.9986 & 13 & 12 & -0.0147 & 0.9657 \\
\hline $\mathrm{CH}_{3} \mathrm{NH}_{2}$ & 9 & 8 & -0.0660 & 0.9985 & 8 & 7 & -0.0148 & 0.9581 \\
\hline
\end{tabular}




\begin{tabular}{|l|l|l|l|l|l|l|l|l|}
\hline $\mathrm{CH}_{3} \mathrm{OH}$ & 9 & 8 & -0.0778 & 0.9981 & 8 & 7 & -0.0137 & 0.9419 \\
\hline $\mathrm{HCOOH}$ & 12 & 11 & -0.0983 & 0.9997 & 11 & 10 & -0.0258 & 0.9580 \\
\hline $\mathrm{CH}_{2} \mathrm{C}(\mathrm{H})(\mathrm{O})^{-}$ & 12 & 11 & -0.0876 & 0.9998 & 10 & 9 & -0.0307 & 0.9570 \\
\hline
\end{tabular}

TABLE 1: Number of positive (pos) and negative (neg) eigenvalues in the Fukui matrix with trace 1 for the molecular set of Otero et al. and most negative eigenvalue (Low). CFMO denotes the coefficient of the relevant FMO in the eigenvector with eigenvalue 1.

From table 1 one immediately sees that the FMO approximation is quite good in most cases. The coefficient of the FMO in the dominant Fukui orbital is usually above 0.9, meaning that it accounts for at least $81 \%$ of the contribution to the Fukui matrix and Fukui function. As expected based on common experience, the FMO is worse for electron attachment, where the FMO corresponds to the LUMO. In some cases the FMO approximation can fail rather badly. The advantage of the Fukui matrix approach is that it directly reveals whether the FMO approximation is good enough whereas previously one had to perform a visual or more complicated numerical comparison between an electron density difference and a FMO density. The Fukui matrix thus flags cases where further analysis based on the FMO approximation might be ill-advised. This is of direct relevance when using the FMO approximation in chemical reactivity studies.

Table 1 also illustrates that the number of positive eigenvalues is always one larger than the number of negative eigenvalues. As there is one eigenvalue equal to 1 within numerical 
accuracy, the number of secondary positive eigenvalues equals the number of negative ones and for all molecules they are paired in the sense mentioned above. This pairing is a consequence of the idempotency of the density matrices considered in this study. It has been shown in general that the eigenvalue spectrum of a matrix that is the difference between two idempotent matrices, should be the same for the negative of this difference, except for possible eigenvalues \pm 1 [33]. For the Fukui matrix, which at the present level of theory corresponds to the difference of two idempotent density matrices, this infers that the spectrum $\sigma$ must fulfill:

$\sigma\left(\mathbf{f}^{ \pm}\right) \backslash\{ \pm 1\}=\sigma\left(-\mathbf{f}^{ \pm}\right) \backslash\{ \pm 1\}$

where $\backslash\{ \pm 1\}$ is used to denote exclusion of these eigenvalues. This is exactly what is observed in the present study and is one of the few cases where this theorem on the difference of idempotent matrices applies in a chemical context. Equation (11) can only hold if all eigenvalues different from 1 or -1 are either zero or come in pairs of values $+\mathrm{x}$ and $-\mathrm{x}$.

The Fukui matrix approach is also useful for addressing the second question: are there regions in space in a molecule where the Fukui function is negative. Regions with negative Fukui functions are counterintuitive since it implies that when an electron is added (removed) to a molecule, some regions of space actually loose (gain) electron density. Negative eigenvalues in the Fukui matrices of the molecules in table 1 are very common. Such eigenvalues are a necessary condition for the occurrence of the much-debated [14-22] negative Fukui function as is immediately clear from equation (10). In general, regions with a negative Fukui function are unlikely because the dominant Fukui orbital overwhelms all other Fukui orbitals in all regions of space. However, in some cases, the dominant Fukui orbital may have small amplitude or even nodes in some regions of space; it is only in these regions that the secondary Fukui orbital pairs 
might play an important role. Even in these regions, there is at least partial cancellation due to the fact that the non-zero eigenvalues come in pairs. To have a negative Fukui function, at least some of the Fukui orbitals with negative eigenvalues must occupy regions in space where the amplitude of the Fukui orbitals with positive eigenvalues is small enough. Unless the Fukui matrix has all three of these properties, negative Fukui functions will not be observed.

The first condition, a low value for the dominant Fukui orbital, will be satisfied near nodal surfaces of this orbital. Note that this was also the reasoning followed by Ayers and coworkers in recent work on the occurrence of negative Fukui functions [20-21]. However, whether a negative Fukui function will appear also depends on the other Fukui orbitals. The Fukui matrix allows for a straightforward analysis of this by inspection of the nodal structure of the dominant Fukui orbital versus the Fukui function minus the contribution of the dominant Fukui orbital in the spectral decomposition (10). For example, one may safely assume that there are no significant regions with negative Fukui functions if none of the secondary Fukui orbitals has a significant occupancy. One can predict, and even understand, the presence of negative Fukui functions by straightforward manipulations of the Fukui matrix and, optionally, visual inspection of the Fukui orbitals using graphical user interfaces. In fact, the entire analysis can be performed very quickly and with less effort than using the more common approach based on comparing functions plotted on some grid. As an example of how the Fukui matrix helps in understanding negative Fukui functions, figure 1 shows for removal of an electron the total Fukui function (a), the sum of its positive contributions (b) and the sum its negative contributions (c) based on the Fukui matrix eigenvalues for FCCF optimized at the B3LYP/6-311++G(2d,p) level and using the corresponding neutral and ionic density matrices. The 0.0002 isosurface of the Fukui function in (a) agrees very well with that reported by Melin et al. [20] in their study of 
negative Fukui functions. However, thanks to the generalization of the Fukui function to the Fukui matrix, one can visualize separately isosurfaces at $|0.0002|$ corresponding to the positive contributions (b) and the negative contributions (c).

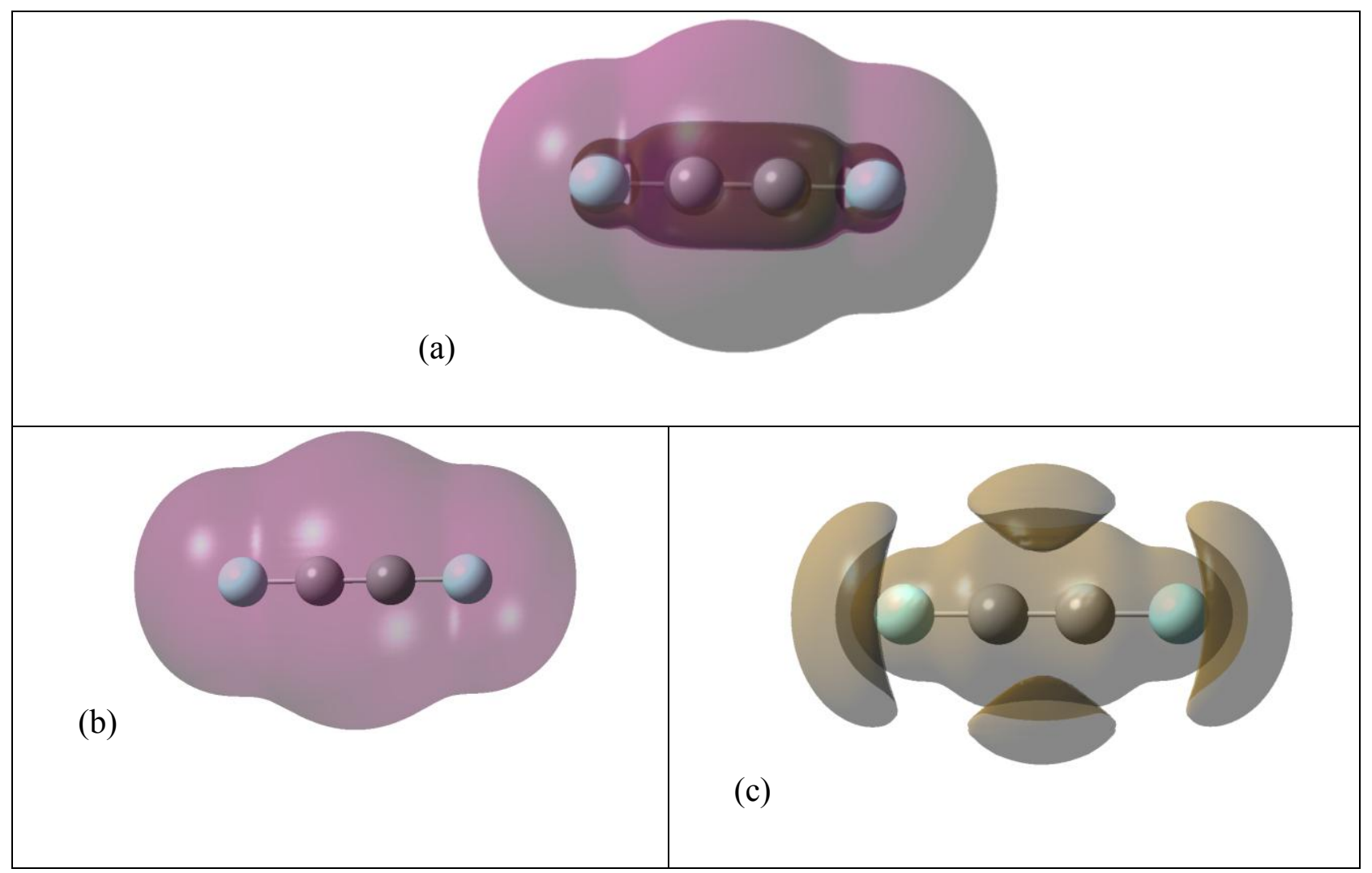

Figure 1: $|0.0002|$ Isosurfaces of $f^{-}(\mathbf{r})$ for the total Fukui function (a), the sum of contributions with positive eigenvalues (b) and negative eigenvalues (c) for FCCF.

The dominant eigenvector consists, in line with the results apparent from table 1, almost entirely from one of the degenerate HOMO orbitals. However, both of these degenerate orbitals has a nodal surface containing the molecular axis while analysis of the positive and negative contributions along this axis reveals that the negative contributions outweigh the positive ones in significantly large regions of space, resulting globally in picture (a) where on the inner part of the molecule the Fukui function results negative (see Melin et al. [20] for a detailed discussion of 
the total Fukui function). This simple example shows that the Fukui matrix reveals that the FMO approximation does not fully account for the Fukui function and reveals at the same time that it can result negative. If so desired, the analysis can be even extended to a Fukui orbital by orbital analysis.

For the practicing chemist this is all valuable information. Even if the FMO approximation breaks down, as happens mainly for cases of addition of an electron, the chemist can still obtain a dominant Fukui orbital that can subsequently be used for chemical interpretation but he/she must take in to account that there are remaining secondary eigenvalues and that any analysis based on the dominant Fukui orbital will fail in regions of space near nodal surfaces of this orbital. At the same time, even though there is always a dominant Fukui orbital, the Fukui matrix explains why regions with negative Fukui functions may occur and where they might be found. This information was not available previously. The Fukui function is still the key quantity for interpreting and even predicting molecular reactivity but the Fukui matrix makes the amount of available information much larger, in the same way that the density matrix is a richer source of information than the density function itself.

\section{CONCLUSIONS}

The Fukui matrix extends the Fukui function in the same way that the density matrix extends the electron density. Specifically, the Fukui function is the diagonal of the Fukui matrix. Using the Fukui matrix, one can dissect the Fukui function into Fukui orbitals and their corresponding eigenvalues. This provides a simple way to assess the quality of the Frontier 
Molecular Orbital (FMO) approximation. It also provides understanding as to whether the Fukui function can be negative in some regions of space and also where a negative Fukui function can be expected. The manipulations required to study the Fukui matrix are very simple algebraic manipulations involving no more than unitary transformations, diagonalization and inversion of matrices.

Tests for a set of organic and inorganic compounds reveal that there is always a dominant eigenvector with an eigenvalue equal to 1 , but that there are numerous other non-zero eigenvalues as well. The latter are found to come in pairs whose eigenvalues have the same magnitude but opposite sign. This is a consequence of the Fukui matrix being a difference of two idempotent density matrices at the level of theory used in this study. The Fukui matrix decomposition provides insight into the occurrence of negative Fukui functions. These will only occur when the dominant eigenvector with eigenvalue 1 is small in a region of space where the eigenvectors with negative eigenvalues are larger than those with positive eigenvalues. By examining the coefficient of the FMO for the dominant Fukui matrix eigenvector, one can assess the quality of the FMO approximation. This approximation is observed to be quite good for electron removal, but less reliable for electron addition.

The extra insights that can be extracted from the Fukui matrix suggest that Fukui function analysis should be routinely performed in this way.

\section{ACKNOWLEDGEMENTS}

P.B. thanks the Fund for Scientific Research in Flanders (FWO-Vlaanderen) for financial support. This work was carried out using the Stevin Supercomputer Infrastructure at Ghent 
University. P.W.A. acknowledges support from NSERC, the Alfred P. Sloan foundation, the Canada Research Chairs, and Sharcnet.

\section{REFERENCES}

1. I. Fleming, Frontier Orbitals and Organic Chemical Reactions, Wiley, New York, 1976.

2. R.G. Pearson, Chemical Hardness - Applications From Molecules to Solids, Wiley-VCH, Weinheim, 1997.

3. P.K. Chattaraj and P.W., Ayers, J. Chem. Phys., 2005, 123, 086101.

4. P.W. Ayers, J. Chem. Phys., 2005, 122, 141102.

5. P.W. Ayers, R.G. Parr and R.G. Pearson, J. Chem. Phys., 2006, 124, 194107.

6. P.W. Ayers, Faraday Discuss., 2007, 135, 161.

7. P.W. Ayers, J .S.M. Anderson and L.J. Bartolotti, Int. J. Quant. Chem., 2005, 101, 520.

8. P. Geerlings, F. De Proft and W. Langenaeker, Chem. Rev., 2003, 103, 1793.

9. R.G. Parr and W. Yang, W., J. Am. Chem. Soc., 1984, 106, 4049.

10. W. Yang, R.G. Parr and R.J. Pucci, J. Chem. Phys., 1984, 81, 2862.

11. J.P. Perdew, R.G. Parr, R. G., M. Levy and J.L. Balduz, Phys. Rev. Lett., 1982, 49, 1691.

12. P.W. Ayers, J. Math. Chem., 2008, 43, 285.

13. P.W. Ayers and M. Levy, Theor. Chem. Acc., 2000, 103, 353.

14. P.W. Ayers, R.C. Morrison and R. K.; Roy, J. Chem. Phys., 2002, 116, 8731.

15. P. Bultinck and R. Carbó-Dorca, J. Math. Chem., 2003, 34, 67.

16. P. Bultinck, R. Carbó-Dorca and W. Langenaeker, J. Chem. Phys., 2003, 118, 4349.

17. P. Senet and M. Yang, J. Chem. Sci., 2005, 117, 411.

18. R.K. Roy, S. Pal and K. Hirao, J. Chem. Phys., 1999, 110, 8236. 
19. R.K. Roy, K. Hirao and S. Pal J. Chem. Phys., 2000, 113, 1372.

20. J. Melin, P.W. Ayers and J.V. Ortiz, J. Phys. Chem. A, 2007, 111, 10017.

21. P.W. Ayers, Phys. Chem. Chem. Phys., 2006, 8, 3387.

22. N. Otero, M. Mandado and R.A. Mosquera, J. Chem. Phys.,2007, 126, 234108.

23. A. J. Coleman and V. I. Yukalov, Reduced Density Matrices: Coulson's Challenge, Springer, New York 2000.

24. P. Hohenberg and W. Kohn, Phys. Rev., 1964, 136,B864.

25. R. McWeeny and B.T. Sutcliffe, Methods of Molecular Quantum Mechanics, Academic Press, London, New York, 1969.

26. R. Balawender and P. Geerlings, J. Chem. Phys., 2005, 123, 124103.

27. T. K. Ghanty, S.K. Ghosh, J. Am. Chem. Soc., 1994, 116, 3943.

28. P. Perez, E. Chamorro and P. W. Ayers, J. Chem. Phys. 2008, 128, 204108.

29. F. De Proft, S. Fias, C. Van Alsenoy and P. Geerlings, J. Phys. Chem. A,2005, 109, 6335.

30. B. Pinter, F. De Proft, V. Van Speybroeck, K. Hemelsoet, M. Waroquier, E. Chamorro, T. Veszpremi and P. Geerlings, J. Org. Chem., 2007, 72,348.

31. X. Feng, J. Yu, R. Liu, M. Lei, W. Fang, F. De Proft and S. Liu, J. Phys. Chem. A, 2010, 114, 6342.

32. Gaussian 03, Revision B.05, M. J. Frisch, G.W. Trucks, H.B. Schlegel, G.E. Scuseria, M.A. Robb, J.R. Cheeseman, J.A. Montgomery, Jr., T. Vreven, K.N. Kudin, J.C. Burant, J.M. Millam, S.S. Iyengar, J. Tomasi, V. Barone, B. Mennucci, M. Cossi, G. Scalmani, N. Rega, G.A. Petersson, H. Nakatsuji, M. Hada, M. Ehara, K. Toyota, R. Fukuda, J. Hasegawa, M. Ishida, T. Nakajima, Y. Honda, O. Kitao, H. Nakai, M. Klene, X. Li, J.E. Knox, H.P. Hratchian, J.B. Cross, V. Bakken, C. Adamo, J. Jaramillo, R. Gomperts, R.E. Stratmann, O. Yazyev, A.J. Austin, R. Cammi, C. Pomelli, J.W. Ochterski, P.Y. Ayala, K. Morokuma, G.A. Voth, P. Salvador, J.J. Dannenberg, V.G. Zakrzewski, S. Dapprich, A.D. Daniels, M.C. Strain, O. Farkas, D.K. Malick, A.D. Rabuck, K. Raghavachari, J.B. Foresman, J.V. Ortiz, Q. Cui, A.G. 
Baboul, S. Clifford, J. Cioslowski, B.B. Stefanov, G. Liu, A. Liashenko, P. Piskorz, I. Komaromi, R.L. Martin, D.J. Fox, T. Keith, M.A. Al-Laham, C.Y. Peng, A. Nanayakkara, M. Challacombe, P.M.W. Gill, B. Johnson, W. Chen, M.W. Wong, C. Gonzalez, J.A. Pople; Gaussian, Inc., Wallingford CT, 2004.

33. R. E. Hartwig and M. S. Putcha, Linear and Multilinear Algebra, 1990, 26, 267. 